\title{
METAL IONS IN BIOLOGICAL SYSTEMS VOLUME 35 IRON TRANSPORT AND STORAGE IN MICROORGANISMS, PLANTS, AND ANIMALS
}

\author{
Edited by Astrid Sigel and Helmut Sigel \\ Marcel Dekker, New York, 1988 \\ 775 pages, ISBN: 0 - 8247 - 9984 - 4
}

Volume 35 of Metal Ions in Biological Systems, entitled Iron Transport and Storage in Microorganisms, Plants, and Animals, offers a comprehensive and timely account of this fascinating topic by 35 distinguished international authorities.

In 18 stimulating chapters, this volume highlights first the biological cycling of iron in oceans, followed by accounts of the transport of iron in microorganisms, fungi, and plants, the roles and properties of siderophores, and the regulation of iron transport and uptake in animals, plants, and microorganisms; it evaluates the structure and structure-function relationships of ferritins and considers their uptake, storage (mineralization), and release of iron; it examines transferrin and its receptor, the homeostasis of iron, especially in humans, and the effect of other metals on iron transport, and it terminates with a chapter about the challenge to create iron chelators for clinical use.

With more than 2200 references to assist further research, Iron Transport and Storage in Microorganisms, Plants, and Animals is an essential resource for scientists and students in many disciplines, including bioinorganic, inorganic, and coordination chemistry; biochemistry; biophysics; molecular biology; enzymology; pharmacology; physiology; clinical chemistry; nutrition; toxicology; and environmental sciences. 